\title{
Activity-Based Restorative Therapies after Spinal Cord Injury: Inter-institutional conceptions and perceptions
}

\author{
David R. Dolbow ${ }^{1 *}$, Ashraf S. Gorgey ${ }^{2,3}$, Albert C. Recio ${ }^{4,5}$, Steven A. Stiens ${ }^{6}$, Amanda C. Curry ${ }^{7}$, Cristina \\ L. Sadowsky, ${ }^{4,5}$, David R. Gater ${ }^{8,9}$, Rebecca Martin ${ }^{4}$, John W. McDonald ${ }^{4,5}$ \\ ${ }^{1}$ University of Southern Mississippi, College of Health, Human Performance and Recreation, Hattiesburg, MS \\ 39406, USA. ${ }^{2}$ Hunter Holmes McGuire VA Medical Center, Spinal Cord Injury and Disorders Center, \\ Richmond, VA 23224, USA. ${ }^{3}$ Virginia Commonwealth University, School of Medicine, Richmond, VA 23298, \\ USA. ${ }^{4}$ Kennedy Krieger Institute, International Center for Spinal Cord Injury, Baltimore MD 21205, USA. \\ ${ }^{5}$ Johns Hopkins University School of Medicine, Baltimore MD 21205, USA. ${ }^{6}$ VA Puget Sound Health Care \\ System, Seattle, WA 98108, USA. ${ }^{7}$ VA Boston Healthcare System, Physical Medicine and Rehabilitation, West \\ Roxbury, MA 02132, USA. ${ }^{8}$ Penn State Hershey Medical Center and Health System, Hershey, PA 17033, USA. \\ ${ }^{9}$ Penn State College of Medicine, Hershey, PA 17033
}

[Received September 12, 2014; Revised October 12, 2014; Accepted November 5, 2014]

\begin{abstract}
This manuscript is a review of the theoretical and clinical concepts provided during an inter-institutional training program on Activity-Based Restorative Therapies (ABRT) and the perceptions of those in attendance. ABRT is a relatively recent high volume and intensity approach toward the restoration of neurological deficits and decreasing the risk of secondary conditions associated with paralysis after spinal cord injury (SCI). ABRT is guided by the principle of neuroplasticity and the belief that even those with chronic SCI can benefit from repeated activation of the spinal cord pathways located both above and below the level of injury. ABRT can be defined as repetitive-task specific training using weight-bearing and external facilitation of neuromuscular activation. The five key components of ABRT are weight-bearing activities, functional electrical stimulation, task-specific practice, massed practice and locomotor training which includes body weight supported treadmill walking and water treadmill training. The various components of ABRT have been shown to improve functional mobility, and reverse negative body composition changes after SCI leading to the reduction of cardiovascular and other metabolic disease risk factors. The consensus of those who received the ABRT training was that ABRT has much potential for enhancement of recovery of those with SCI. Although various institutions have their own strengths and challenges, each institution was able to initiate a modified ABRT program.
\end{abstract}

Key words: Neuroplasticity, Massed practice, Locomotor Training, Task-specific Practice, Activity-based Restorative Therapies

In June of 2012, representatives from three wellestablished rehabilitation centers participated in a weeklong intensive inter-institutional training course on Activity-Based Restorative Therapies (ABRT). ABRT, a term developed over the last decade, describes a relatively recent high volume and intensity approach to the deficits induced by neurological paralysis [1]. Although this approach to neuro-rehabilitation is intended to treat a wide array of neurological conditions, its development is rooted in research conducted in spinal cord injury (SCI) rehabilitation. For individuals with $\mathrm{SCI}$, the goal of ABRT is to provide activation of the spinal cord pathways located above and below the level of injury through a variety of rehabilitation interventions [1]. This increase in neural activity is thought to be beneficial toward the restoration of non-functioning

*Correspondence should be addressed to: Dr. David Dolbow, University of Southern Mississippi, 118 College 
neural pathways and toward the promotion of new connections and reconnecting of former connections $[1,2]$.

\section{Basic Science: Philosophy and Origin of ABRT}

The most obvious result of SCI is spinal paralysis. Spinal paralysis is defined as the loss of feeling and/or movement caused by damage to the spinal cord [3]. Damage to the spinal cord may result in a number of challenges making restoration of function difficult. These challenges include: (A) damaged glial cells that formulate a glial scar blocking transmission of neural messages, (B) demyelination of neural axons which results in the breakdown of synaptic interaction with intact neurons distal to the injury site, and (C) inhibitory molecules produced by the body that interfere with regrowth of neural axons [4]. These factors often result in a formidable glial scar encased oval-shaped lesion spanning several spinal levels with axonal demyelination throughout the area (Figure 1). While investigative treatments continue to develop avenues to breakdown glial scars and to block inhibitory molecules, it is theorized that ABRT can be utilized to promote myelination of the endogenous cells and encourage sprouting of new collateral axons [1] and increase birth of tripotential progenitor cells [5]. Additionally, ABRT is thought to promote spinal learning or central pattern generation with task specific training $[1,2]$.

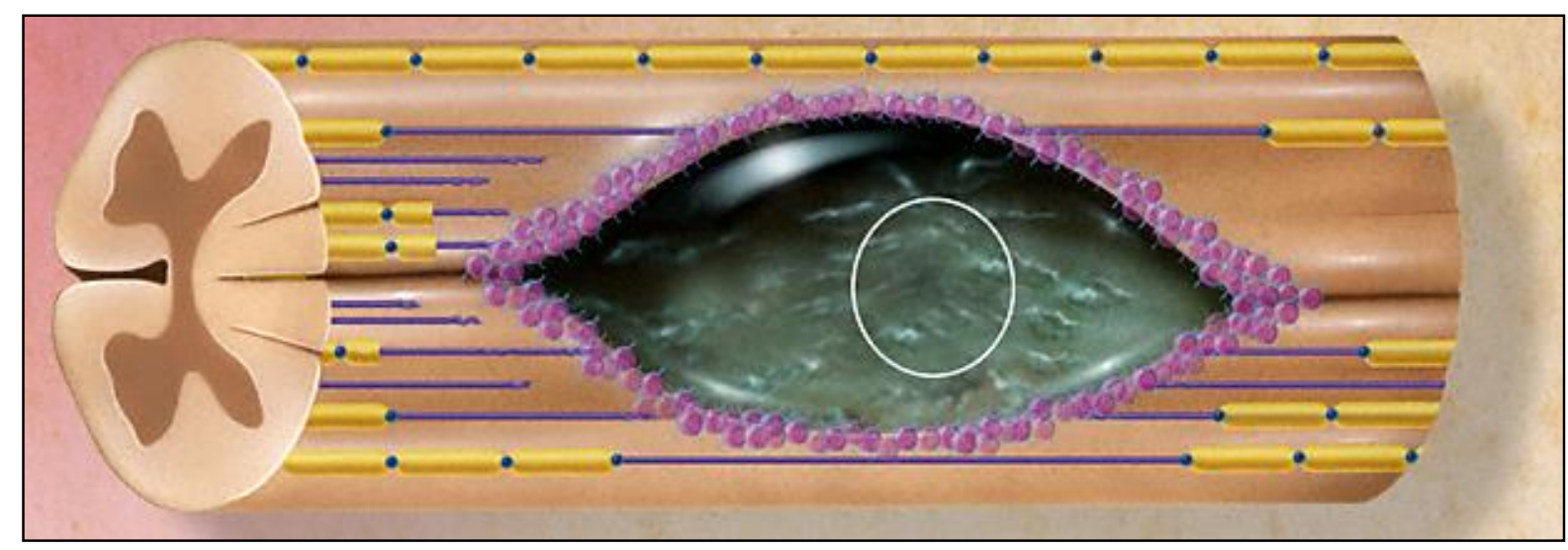

Figure 1. Spinal cord lesion with glia scarring and axon demyelination.

The purpose of this intensive training course was to develop and introduce an educational program on the principles and clinical practice of ABRT. The purpose of this paper is to provide a historical description of the theoretical and clinical development of ABRT, provide a comprehensive description of the requirements of an ABRT program and present information regarding the initial implementation of the programs at each facility that attended the training course.

The primary principles and modalities that make up ABRT have a long history involving rehabilitation and research of spinal cord conditions. Over a century ago, Sherrington et al. promoted the thought that walking is controlled by a succession of peripheral reflexes [6]. Grillner and Rossignol provided support for this idea by demonstrating that when the hip joint of spinally transected cats is sufficiently extended, the muscle spindles of the hip flexor muscles are initiated facilitating a stepping motion [7].

Later, Lovely and colleagues demonstrated that spinally transected cats could regain the ability to walk after training on a treadmill with partial weight-bearing and manual assistance facilitating the stepping of the hind legs [8]. Transferring this idea to humans, Harkema et al. provided evidence that individuals with and without SCI have increased hip extension activation with increased weight-bearing [9]. With increased activation of hip extension comes a greater capacity for the reciprocal motion of hip flexor and forward stepping. Dietz and associates verified the importance of weightbearing by showing that humans with paraplegia and tetraplegia display no muscle activation with passive non-weight-bearing movement of the legs [10].

Hodgson et al. introduced another primary component of ABRT by showing the importance of task- 
specific training [11]. In their study, spinally transected cats improved the ability to walk but not stand with ambulation training, and improved the ability to stand but not walk with standing training. Thus, over the last century numerous studies have supported the theory that the neuronal circuitry responsible for generating an efficient stepping pattern in non-human mammals is embedded within the spinal cord [12]. This appears to also be true for humans, although the development of the stepping pattern with training has not reached the same levels of restoration. Nevertheless, the spinal locomotor centers of humans with complete and incomplete SCI can be activated by appropriate afferent input and individuals with incomplete SCI have shown substantial benefit from ABRT training [13].

The principles of training used in ABRT have been established upon the results of these and other research studies and can be summarized as repetitive task-specific training using weight-bearing and external facilitation of neuromuscular activation.

Although the origin of ABRT involves many factors spanning a century or more, the rehabilitation process of Christopher Reeve is an easily identifiable recent event that caused a major shift in the rehabilitation paradigm. Christopher Reeve, well known for his portrayal as "Superman" in motion-pictures, suffered fractures to the first and second cervical vertebrae during an equestrian accident. This event, which occurred in 1995, resulted in a C2 American Spinal Injury Association Impairment Scale (AIS) A, as per International Standards for Neurological Classification of Spinal Cord Injury (ISNCSCI). This left Mr. Reeve with complete motor and sensory loss below the level of injury (www.medscape.com/viewarticle/491399) [14].

Mr. Reeve's rehabilitation program included positioning, passive range of motion exercises and functional electrical stimulation (FES) cycling in order to decrease the effects of accelerated aging and occurrence of secondary complications associated with SCI such as pressure ulcers, joint contractures and pneumonia. However, Mr. Reeve also unexpectedly regained voluntary movement of his left index finger. Although this was a relatively small gain physically, it sparked a paradigm shift in rehabilitation philosophy (www.medscape.com/viewarticle/491399).

Encouraged by this development five years postinjury, the rehabilitation team designed a more aggressive program of physical activity including functional electrical stimulation lower-limb cycling and aquatic therapy in order to increase patterned neural activity [14]. Unfortunately, Mr. Reeve passed away in 2004 due to medical complications unrelated to ABRT. However, prior to Mr. Reeve's passing, he reportedly regained greater than $70 \%$ sensation and more than $20 \%$ motor ability below the level of injury (www.medscape.com/viewarticle/491399); www.healingtherapies.info/Aggressive-Rehab2htm)

[14]. The significant restoration of sensation and motor function had heretofore been thought improbable for someone with a chronic SCI, thus creating a change in the theory of recovery for those with SCI.

\section{Important Physiological Principles}

The principle of neuroplasticity is the bedrock of the ABRT philosophy. Prior to the development of the neuroplasticity principle, healthcare providers practiced under the assumption that the central nervous system was hardwired and that functional loss due to damage in the spinal cord or brain could not be recovered. The rehabilitation focus concentrated on compensation for the loss rather than restoration [15].

However, with the advent of the principle of neuroplasticity, healthcare providers have gradually moved toward the belief that intact but damaged spinal pathways and neural synapses may undergo restorative changes and that new connections may be developed through repetitive physical activities many years after injury $[1,2,15]$. While neuroplasticity had its original focus in the brain, this same principle provides impetus for rehabilitation of those with SCI. Neuroplasticity is defined as the capacity of the nervous system to change connections and behavior in response to new information and stimulation (www.merriam-webster.com/dictionary /neuroplasticity).

While neuroplasticity is a main principle of focus for ABRT, other physical activity driven physiological principles are also heavily involved. The "Over-load principle" is perhaps the most well-known principle relating to exercise and training. A general definition of this principle is that the body adapts to workload placed upon it; thus, this principle determines the size and strength of muscles and helps in determining the functional capacity of the cardiovascular system [16]. Likewise, "Wolf"s law" and its derivative "The Mechanostat theory", asserts that bone strength adapts to mechanical loads with the greatest caused by muscle contractions [17]. Loss of muscle activity and the associated positive effects on the muscular, skeletal and cardiovascular systems results in negative effects on all three systems. Reportedly, after SCI, the average crosssectional area of sub-lesional skeletal muscle decreases by as much as $45-80 \%$ [18]. Similarly, after SCI, there is a decrease of up to $33 \%$ of bone mass below the level of injury within the first three to four months and up to $50 \%$ within the first 3 years [18-25]. When SCI results in loss of input from the sympathetic nervous system (T6 and above), bradycardia, bradyarrhythmia and hypotension 
often result [26]. The combination of impaired autonomic control, decreased musculature available for exercise, sedentary lifestyle and the resultant decreased cardiovascular fitness increases the risk of cardiovascular disease which has emerged as a leading cause of death for those with SCI [27]. There is also consistent information indicating that there is a higher prevalence of cardiovascular disease (CVD) after SCI in comparison to the ambulatory population. For instance, the prevalence rates of symptomatic CVD has been approximated to be $30-50 \%$ for those with SCI while in the general able-bodied population the prevalence is approximately $5-10 \%[28,29]$. In addition, asymptomatic prevalence of CVD has been reported to be $60-70 \%$ in persons with SCI [30,31]. These are alarming statistics that place significant burden upon the patient, family, and society as a whole.

A third component of body composition, fat, is also typically affected by SCI. The "Energy balance principle" states that the body's energy storage (adipose tissue) is guided by the formula (changes in energy store = energy intake - energy expenditure). Thus, with decreased physical activity after SCI, fat mass (FM) and percent body fat mass increase. Percent body fat frequently reaches $30 \%$ or more and is associated with an average $58 \%$ greater cross-sectional area of visceral adipose tissue in those with SCI compared to age and waist circumference-matched able-bodied individuals [32].

Therefore, there is ample evidence that body composition changes play a major role in negatively affecting the health of the individuals with SCI. It is fairly obvious that dramatic bone loss after SCI can lead to neurogenic osteoporosis and an increased risk of fractures [22,33]. Reportedly, following SCI, 14\% of individuals will have fractures in the first five years, $28 \%$ within ten years, and $39 \%$ within 15 years of injury [34]. It is also obvious that individuals with SCI-related paralysis have significantly decreased muscle mass and increased FM. Increased adipose tissue and decreased muscle mass have been closely related to an increased risk of coronary artery disease and metabolic syndrome which is often a precursor to diabetes mellitus. Metabolic syndrome includes reduced glucose tolerance, hyperinsulinemia, dyslipidemia, elevated blood pressure and waist circumferences greater than 40 inches in males. Dyslipidemia is characterized by elevated triglyceride, elevated low-density lipoprotein levels and depressed high-density lipoprotein cholesterol levels, with the latter being the characteristic dyslipidemic change in individuals with SCI related paralysis and an independent risk factor for development of CVD $[32,34,35]$.

\section{Medical Management}

The medical management of individuals with SCI is vital during a program of ABRT. This process includes evaluation and the monitoring of several organ systems affected by paralysis for a variety of conditions. In order to ensure the safety of the participants, initial medical examination and subsequent management includes screening for autonomic dysreflexia, deep venous thrombosis, osteoporosis, urinary tract infections, respiratory infections including pneumonia and soft tissue injuries i.e., pressure ulcers and musculo-skeletal overuse injuries. These screens are very important because any single or combination of these problems could have devastating consequences on long-term health and independence. A complete neurological examination including an assessment of sensory and motor function is performed initially and periodically during the program. This is important for the initial development of a personalized ABRT program and to document changes throughout the program.

\section{Key Therapeutic Components of ABRT}

As with all comprehensive rehabilitation programs, exercise designed to develop joint range of motion and muscle strength are incorporated into all phases of the ABRT program and are especially essential for the initiation of weight bearing activities. The five key therapeutic components of ABRT are (A) weight-bearing activities, (B) FES, (C) task-specific practice, (D) massed practice, and (E) locomotor training, all applied in the context of optimal neuro-stimulation above and below neurologic injury level.

Weight-bearing activities include a wide variety of activities using all four limbs individually or in combination. These activities include tall kneeling, weight bearing on arms while seated (sides, front and back), quadruped (all four limbs), standing using frames or other supportive devices, and a variety of locomotor activities. Weight-bearing activities have not only been shown to slow the loss of bone during the acute stage of SCI [35] and decrease extensor muscle spasms [36], but also to provide a wide range of benefits imperative to overall health $[37,38]$. Walters and associates reported that standing for 30 minutes or more per day has been shown to significantly improve quality of life, decrease incidents of pressure ulcers, decrease bladder infections, improve bowel regularity and the ability to straighten one's legs [39].

FES includes upper- and lower- limb cycling and resistive exercise training via electrical stimulation of individual muscle groups. FES can also be utilized to assist movements during gait training, standing, 
transferring and various other upper and lower limb activities. Some of the more obvious benefits of FES activity include the improvement of body composition through the increase in lean mass (LM), decrease in FM and increase in bone mineral density (BMD) below the level of injury [39,40-42]. In turn, the positive body composition changes reduce the risk of cardiovascular and metabolic diseases [43]. FES of the lower-limbs, particularly repetitive FES cycling, has been reported to increase stroke volume $[44,45]$ and help reverse myocardial disuse atrophy $[45,46]$. There is also growing evidence from several trials that FES training for a minimum of three days per week for two months or more can improve oxidative metabolism [47,49], exercise tolerance [45,50-52] and cardiovascular fitness $[45,47,48,50,52,53]$. An additional possible benefit of FES is the reduced risk of pressure ulcers through increased gluteal muscle hypertrophy and associated increased vascularization $[54,55,56]$.

One of the more encouraging benefits of FES is the incremental cellular changes that appear to be possible in the nervous system (2). Rodent studies using electrical stimulation have produced the birth of tripotential endogenous neural progenitor cells in the spinal cord [5,57]. A large number of these cells have developed into myelin producing oligodendrocytes which provides great hope for the restoration of axonal re-myelination of damaged neurons [5,57]. Overall, whether it be through neural or body composition alterations, regular FES activity has been shown to increase the quality of life of those with SCI [58].

Task-specific practice is thought to be imperative to the development of skilled movements. The key to learning any skill begins with repetition of the same movement over and over to develop the motor pattern. This component of the ABRT program is driven by the goals of the participant and the rehabilitation team. Because functional restoration has been shown to be at least partially dependent on task specific training [10] functional activities that relate to these goals are included in the program, i.e., walking, transfers, and bed mobility.

Massed practice is a vital component of the program as the number of repetitions and the extended amount of activity time is thought to be crucial in initiating positive results in the spinal cord whether it be stimulating remyelination or developing new neural connections. The program consists of up to five hours of therapy daily. This time may include the skilled physical therapy and occupational therapy training sessions, restorative exercise with technicians or other therapy professionals trained for specific activities such as FES cycling, water treadmill training and patient self-exercise, which may include a planned and well-rehearsed home rehabilitation program. Massed practice is altered as the patient is able to tolerate the activities. The order of activities, skilled monitoring of the participants abilities, and well-timed rest breaks are important to the success of the massed practice component.

The fifth vital component of ABRT is locomotor training which includes BWSTT during which the participant is secured from an overhead support via an upper-body harness and trained on a moving treadmill. During BWSTT, the participant ambulates while trained staff members facilitate the movements of each leg while stabilizing the trunk. Each staff member is specially trained so that hand placements over specific muscle tendons and muscle bodies help to facilitate muscle activation, while assisting with the completion of the desired activity. All trainers coordinate their actions with the actions of the other trainers and the participant in order to result in the skilled ambulatory activity. The speed of the treadmill and the amount of weight bearing during the activity is determined and controlled by trained staff. In addition to promoting the restoration of walking via neuroplasticity $[13,59]$, BWSTT has been shown to increase maximum voluntary muscle contractions by $28-34 \%$ and decrease spasticity [60].

BWSTT is also conducted on an underwater treadmill in a therapeutic pool, although instead of a mechanical harness, water buoyancy decreases the amount of weight bearing depending on the depth of the water. For this activity, ABRT staff members are trained to utilize their feet and legs as well as their hands and arms in order to facilitate the participant's leg movements under water. Standing and over-ground gait training are also utilized in order to provide practical training leading to functional community ambulation.

\section{Practical Application}

Because of the devastating nature of SCI, a primary focus of the ABRT team is to remain positive and enthusiastic toward the tasks at hand and promoting achievement of the rehabilitation goals. The radiation of this attitude and demeanor toward the patients encourages high morale and determination. This is possible because of the growing body of evidence supporting the benefits of ABRT.

The host training facility demonstrated a wide variety of ABRT activities including supported standing, BWSTT, water treadmill training, FES-assisted ambulation, over-ground ambulation training with a variety of assistive devices, FES cycling, weight bearing activities with all limbs on the mat, bed mobility and transfer training with manual and/or electrical stimulation assistive techniques, and muscle strengthening facilitated manually and with electrical 
stimulation. Games were also incorporated into the therapy sessions for younger patients and at times patients and therapists teamed up for friendly competition during therapy sessions to add further encouragement and fun during the therapy process.

\section{Interpretations}

The prevailing opinion of the course participants was that ABRT has a potential for enhancing the recovery of those with SCI. However, a primary limitation is that most medical centers are unable to match the trained staffing and equipment resources of the host facility. Staffing in most rehabilitation programs is not adequate to provide three to five hours of skilled therapy for each patient. Furthermore, most medical centers do not have the equipment to provide BWSTT or underwater treadmill training. Nevertheless, there was a consensus of thought that the ideas promoted during the training could be introduced at most medical centers, albeit on a smaller scale, with graded and gradual introduction.

One idea that was embraced was the introduction of using small portable inexpensive electrical stimulation units to promote strengthening of various muscle groups while patients are performing other typical therapeutic activities. For example, incorporating portable neuromuscular electrical stimulation to upper-limb muscles for the purpose of assisting with the grasping and moving an object or simply using electrical stimulation to complete muscle contractions with lowerlimb muscle groups while simultaneously working on skilled upper-limb activities. This allows muscle strengthening to occur in the lower limbs simultaneously with upper-limb rehabilitation.

With each rehabilitation center having its own particular strengths and limitations, each program proceeded in its own way and at its own pace. The three rehabilitation centers were able to successfully initiate a modest ABRT program including FES cycling and task specific practice. Locomotor training was also incorporated into the three programs, although only two of the facilities had the equipment and staffing to initiate BWSTT. One facility was able to utilize research staff members that were already trained in the techniques of BWSTT, while the facility that was unable to initiate BWSTT reported that, although staff members were interested and willing, the required $\geq 3$ to 1 ratio of staff to patient was difficult to manage. In line with ABRT's focus on long term participation in physical activities, one facility was able to utilize home-based FES cycling for continuation of the ABRT program. After sixteen weeks, the exercise adherence of 17 individuals with SCI remained at $63 \%$, which is essentially twice the exercise rate of the able-bodied population in the U.S. [38].
Because all three rehabilitation centers serve a population that is different than that of the training institution, the ABRT programs are necessarily different. For example, even though younger adults are also treated, the average age of the individuals with SCI enrolled in rehabilitation is typically older, with many in their fifth through eighth decades of life and often with co-morbidities. Also, a large number of the participants at these facilities were in the acute phase of injury, limiting out of bed tolerance. It is also not unusual for patients to have poor family and social supports complicating discharges and home rehabilitation programs.

The general consensus of the ABRT course participants was that each rehabilitation center had its own set of strengths and challenges; thus, each program will need to be individualized. However, even with individual facility limitations, each program has been able to initiate a modified form of ABRT which enhanced enthusiasm for this emerging approach to neuro-rehabilitation.

\section{Declaration of Interest}

The ABRT Inter-Institutional training program held at the International Center for Spinal Injury at Kennedy Krieger Institute was funded by a grant from the Department of Defense. Financial disclosure statements have been obtained, and no conflicts of interest have been reported by the authors or by any individuals in control of the content of this article.

\section{References}

[1] Sadowsky CL, McDonald JW (2009). Activity-based restorative therapies: concepts and applications in spinal cord injury-related neurorehabilitation. Dev Disabili Res Rev, 15(2):112-116.

[2] Martin R, Sadowsky C, Obst K, Brooke M, McDonald J (2012). Functional Electrical Stimulation in Spinal Cord Injury: From Theory to Practice. Top Spinal Cord Inj Rehabil, 18(1):18(1): 28-33.

[3] Bear MF, Connors BW, Paradiso MA (2007). Neuroscience: past, present, and future. In Neuroscience: Exploring the Brain. 3rd ed. Lippincott Williams \& Wilkins, P19.

[4] Fitch MT, Silver J (2008). CNS injury, glial scars, and inflammation: Inhibitory extracellular matrices and regeneration failure. Exp Neurol, 209(2): 294-301.

[5] Becker D, Gary DS, Rosenzweig ES, Grill WM, McDonald JW (2010). Functional electrical stimulation helps replenish progenitor cells in the injured spinal cord of adult rats. Exp Neurol, 222(2):211-8.

[6] Sherrington CS (1910). Flexion-reflex of the limb, crossed extension-reflex, and reflex stepping and standing. J Physiol, 40(1-2):28-121. 
[7] Grillner S, Rossignol S (1978). On the initiation of the swing phase of locomotion in chronic spinal cats. Brain Res, 146(2):269-277.

[8] Lovely RG, Gregor RJ, Roy RR, Edgerton RV (1986). Effects of training on the recovery of full-weightbearing stepping in the adult spinal cat. Exp Neurol, 92(2):421-435.

[9] Harkema SJ, Hurley SL, Patel UK, Dobkin BH, Edgerton VRT (1997). Human lumbrosacral spinal cord interprets loading during stepping. J Nuerophysiol, 77(2):797-811.

[10] Dietz V, Mueller R, Colombo G (2002). Locomotor activity in spinal man: significance of afferent input from joint and load receptor. Brain, 125:2626-2634.

[11] Hodgson JA, Roy RR, De Leon R, Dobkin B, Edgerton VR (1994). Can the mammalian lumbar spinal cord learn a motor task. Med Sci Sports Exerc, 26:14911497.

[12] Hubli M, Dietz V (2013). The physiological basis of neutorehabilitation-locomotor training after spinal cord injury. J Neuroeng Rehabil, Jan 21,10:5

[13] Dietz V (2008). Body weight supported gait training: from laboratory to clinical setting. Brain Res Bull, July 30 76(5):459-463.

[14] McDonald JW, Becker D, Sadowsky CL, Jane JA Sr, Conturo TE, Schultz LM (2002). Late recovery following spinal cord injury; case report and review of the literature. Journal NeurosurgerySpine, (97):252265.

[15] Behrman AL, Bowden MG, Nair PM (2006). Neuroplasticity after spinal cord injury and training: an emerging paradigm shift in rehabilitation and walking recovery. Phy Ther, 86(10):1406-1425.

[16] Sanders M, Sanders B (2001). Principles of Resistance Training in Therapeutic Exercise: Techniques for Intervention, Ed. Bandy WD, Sanders B.; Lippincott Williams \& Wilkins. Philadelphia. PA. Pg 91.

[17] Frost HM (1987). Bone "mass" and the "mechanostat": A proposal. Anat Rec, 219(1):1-9.

[18] Castro JM, Apple DF Jr., Hillegass EA, Dudley GA (1999). Influence of complete spinal cord injury on skeletal muscle cross-sectional area with the first 6 months of injury. Eur J Appl Physiol Occup Phsyiol, 80:373-378

[19] Broholm B, Podenphant J, Biering-Sorensen F (2005). The course of bone mineral density and biochemical markers of bone turnover in early postmenopausal spinal cord-lesioned females. Spinal Cord, 43:674-677.

[20] Dauty M, Perrouin Verbe B, Maugars Y, Dubois C, Mathe JF (2000). Supralesional and sublesional bone mineral density in spinal cord-injured patients. Bone, 27(2):305-309.

[21] Dudley-Javoroski S, Shields RK (2008). Muscle and bone plasticity after spinal cord injury: Review of adaptations to disuse and to electrical muscle stimulation. J Rehabil Res Dev, 5(2):283-296.

[22] Eser P, Frotzler A, Zehnder Y, Wick L, Knecht H, Denoth J, Schiessl H (2004). Relationship between the duration of paralysis and bone structure: a pQCT study of Spinal cord injured individuals. Bone, 34(5):869880.

[23] Garland DE, Stewart CA, Adkins RH, Hu SS, Rosen C, Liotta FJ, Weinstein DA (1992). Osteoporosis after spinal cord injury. J Orthop Res, 10(3):3871-3878.

[24] Sheilds RK (2002). Muscular, skeletal, and neural adaptations following spinal cord injury. J Orthop Sports Phys Ther, 32(2):65-74.

[25] Sniger W, Garshick E (2002). Alendronate increases bone density in chronic spinal cord injury: A case report. Arch Phys Med Rehabil, 83(1):139-140.

[26] Furlan JC, Fehlings MG (2008). Cardiovascular complications after spinal cord injury: pathophysiology, diagnosis, and management. Neurosurg Focus, 25(5): E131

[27] Bauman AW, Spungen AM (2008). Coronary heart disease in individuals with spinal cord injury: assessment of risk factors. Spinal Cord, 46(7):466-76.

[28] Groah SL, Weitzenkamp D, Sett P, Soni PB, Savic G (2001). The relationship between neurological level of injury and symptomatic cardiovascular disease risk in the aging spinal injured. Spinal Cord, 39(6):310-317.

[29] Myers J, Lee M, Kiratli J (2007). Cardiovascular disease in spinal cord injury: an overview of prevalence, risk, evaluation, and management. Review. Am J Phys Med Rehabil, 86(2):142-152.

[30] Bauman WA, Raza M, Chayes Z, Machac J (1993). Tomographic thallium-201 myocardial perfusion imaging after intravenous dipyridamole in asymptomatic subjects with quadriplegia. Arch Phys Med Rehabil, 74(7):740-4.

[31] Bauman WA, Spungen AM (1994). Disorders of carbohydrate and lipid metabolism in veterans with paraplegia or quadriplegia: a model of premature aging. Metabolism, 43(6):749-56.

[32] Edwards LA, Bugaresti JM, Bucholtz AC (2008). Visceral adipose tissue and the ratio of visceral to subcutaneous adipose tissue are greater in adults with spinal cord injury than those without spinal cord injury, despite matching waist circumferences. Am JClin Nutr, 87(3):600-607.

[33] Sorensen FB, Bohr H, Schaadt O (1998). Bone mineral content of the lumbar spine and lower limbs years after spinal cord lesion. Paraplegia, 26:293-301.

[34] Maynard FM, Karunas RS, Adkins RH (1995). Management of the neuromuscular system. In: Stover SL, Delisa JA, Whiteneck GG. ed. Spinal cord injury: clinical outcomes of the model systems. Aspen, Gaitersburg, MD. p163-169.

[35] Bauman WA, Spungen AM, Wang J, Pierson RN Jr (2004). The relationship between energy expenditure and lean tissue in monozygotic twins discordant for spinal cord injury. J Rehabil Res Dev, 41(1):1-8.

[36] de Bruin ED, Frey-Rindova P, Herzog RE, Dietz V, Dambacher MA, Stussi E (1999). Changes of tibia bone properties after spinal cord injury: effects of early intervention. Arch Phys Med Rehabil, 80(suppl 2):214220 .

[37] Adams MM, Hicks AL (2011). Comparison of effects of body-weight-supported treadmill training and tilt- 
table standing on spasticity in individuals with chronic spinal cord injury. J Spinal Cord Med, 34(5):488-94.

[38] Dolbow DR, Gorgey AS, Ketchum JM, Moore JR, Hackett LA, Gater DR (2012). Exercise adherence during home-based functional electrical stimulation cycling by individuals with spinal cord injury. Am J Phys Med Rehabil, 91(11):922-930.

[39] Walter JS, Sola PG, Sacks J, Lucero Y, Langbein E, Weaver F (1999). Indications for a home standing program for individuals with spinal cord injury. J Spinal Cord Med, 22(3):152-158.

[40] Frotzler A, Coupaud S, Perret C, Kakebeeke TH, Hunt KJ, Donaldson Nde N, Eser P (2008). High-volume FES-cycling partially reverses bone loss in people with chronic spinal cord injury. Bone, 43:169-176.

[41] Gorgey AS, Mather KJ, Cupp HR, Gater DR (2012). The effects of resistance training on adiposity and metabolism after spinal cord injury. Med Sci Sports Exerc, 1:165-174.

[42] Dolbow JD, Dolbow DR, Gorgey AS, Adler RA, Gater DR (2013). The effects of aging and electrical stimulation exercise after spinal cord injury. Aging Dis, 4(3):141-153.

[43] Griffin L, Decker MJ, Hwang JY, Wang B, Kitchen K, Ding Z, Ivy JL (2009). Functional electrical stimulation cycling improves body composition, metabolic and neural factors in persons with spinal cord injury. $\mathbf{J}$ Electromyogr Kinesiol, 19(4):614-622.

[44] Gater DR Jr (2007). Obesity after spinal cord injury. Phys Med Rehabil Clin N Am, 18(2):333-351.

[45] Hooker SP, Figoni SF, Rodgers MM, Glaser RM, Mathews T, Suryaprasad AG, Gupta SC (1992). Physiologic effects of electrical stimulation leg cycle exercise training in spinal cord injured persons. Arch Phys Med Rehabil, 73(5):470-476.

[46] Nash MS, Bilsker S, Marcillo AE, Isaac SM, Botelho LA, Klose KJ, Green BA, Rountree MT, Shea JD (1991). Reversal of adaptive left ventricular atrophy following electrically-stimulated exercise training in human tetraplegics. Paraplegia, 29(9):590-599.

[47] Andersen JL, Mohr T, Biering-Sorensen F, Galbo H, Kjaer M (1996). Myosin heavy chain isoform transformation in single fibres from $\mathrm{m}$. vastus lateralis in spinal cord injured individuals: effects of long-term functional electrical stimulation (FES) Pflugers Arch, 431(4):513-518.

[48] Brurok B, Torhaug T, Karlsen T, Leivseth G, Helgerud J, Hoff J (2013). Effect of lower limb functional electrical stimulation pulsed isometric contractions on arm cycling peak $\mathrm{O} 2$ uptake in spinal cord injury individuals. J Rehabil Med, 45(3):254-259.

[49] Mohr T, Andersen JL, Biering-Sorensen F, Galbo H, Bangsbo J, Wagner A, Kjaer M (1997). Long-term adaptation to electrically induced cycle training in severe spinal cord injured individuals. Spinal Cord, 35(1):1-16.

[50] Barstow TJ, Scremin AM, Mutton DL, Kunkel CF, Cagle TG, Whipp BJ (1996). Changes in gas exchange kinetics with training in patients with spinal cord injury. Med Sci Sports Exerc, 28(10):1221-1228.

[51] Crameri RM, Cooper P, Sinclair PJ, Byrant G, Weston A (2004). Effect of load during electrical stimulation training in spinal cord injury. Muscle Nerve, 29(1):104111.

[52] Pollack SF, Axen K, Spielholz N, Levin N, Haas F, Ragnarsson KT (1989). Aerobic training effects of electrically induced lower limb exercises in spinal cord injured people. Arch Phys Med Rehabil, 70(3):214219.

[53] Thijssen DH, Heesterbeek P, van Kuppevelt DJ, Duysens J, Hopman MT (2005). Local vascular adaptations after hybrid training in spinal cord-injured subjects. Med Sci Sports Exerc, 37(7):1112-1118.

[54] Dolbow DR, Gorgey AS, Dolbow JD, Gater DR (2013). Seat pressure changes after eight weeks of functional electrical stimulation cycling: A pilot study. Top Spinal Cord Inj Rehabil, 19(3):222-228.

[55] Petrofsky JS (1992). Functional electrical stimulation, a two year study. J Rehabil, 58(3):29-34.

[56] Hjeltnes N, Aksnes AK, Birkeland KI, Johansen J, Lannem A, Wallberg-Henriksson H (1997). Improved body composition after 8 wk of electrically stimulated leg cycling in tetraplegic patients. Am J Physiol, 273(3 Pt 2):R1072-1079.

[57] Li Q, Brus-Ramer M, Martin JH, McDonald JW (2010). Electrical stimulation of the medullary pyramid promotes proliferation and differentiation of oligodendrocyte progenitor cells in the corticospinal tract of the adult rat. Neurosci Lett, 479(2):128-133.

[58] Dolbow DR, Gorgey AS, Ketchum JM, Gater DR (2013). Home-based functional electrical stimulation cycling enhances quality of life in individuals with spinal cord injury. Top Spinal Cord Inj Rehabil, 19(4):324-329.

[59] Dietz V (2012). Neuronal plasticity after a human spinal cord injury: positive and negative effects. Exp Neurol, 235(1):110-115.

[60] Gorgey AS, Poarch H, Harnish C, Miller JM, Dolbow D, Gater DR. (2011). Acute effects of locomotor training on neuromuscular and metabolic profile after incomplete spinal cord injury. NeuroRehabilitation, 29(1):79-83. 